\title{
Role of OmpA and IbeB in Escherichia coli K1 Invasion of Brain Microvascular Endothelial Cells In Vitro and In Vivo
}

\author{
YING WANG AND KWANG SIK KIM \\ Division of Infectious Diseases, Childrens Hospital Los Angeles, Los Angeles, California 90027, U.S.A. \\ [Y.W., K.S.K.], and Division of Pediatric Infectious Diseases, Johns Hopkins University School of \\ Medicine, Baltimore, Maryland 21287, U.S.A. [K.S.K.]
}

\begin{abstract}
Escherichia coli $\mathrm{K} 1$ is the most common Gram-negative organism causing neonatal meningitis, but it is incompletely understood how E. coli $\mathrm{K} 1$ crosses the blood-brain barrier. We have previously identified several $E$. coli determinants contributing to invasion of brain microvascular endothelial cells (BMEC) in vitro, which include OmpA and IbeB. In the present study, we constructed a mutant (E98) by deleting only OmpA (isogenic OmpA deletion mutant) from E. coli K1 strain RS218 (018:K1:H7) and also an isogenic OmpA deletion mutant from the $i b e B$-deleted mutant (IB7D5) of strain RS218. As expected, the ompA and $i b e B$ deletion mutants, E98 and IB7D5, respectively, were less invasive in BMEC in vitro compared with the parent strain. More importantly, their abilities to penetrate the blood-brain barrier were significantly less than those of the parent strain in the experimental hematogenous $E$. coli meningitis model. The combined ompA- and $i b e B$-deleted mutant, however, behaved similarly compared with its single-gene deletion mutants (E98 and IB7D5) in its ability to invade BMEC in vitro
\end{abstract}

\section{ABSTRACT}

and to penetrate into the CNS in vivo. These findings indicate that OmpA and IbeB are the important determinants contributing to E. coli $\mathrm{K} 1$ crossing of the blood-brain barrier, but their contributions are not additive. Additional studies are needed to understand the reasons for no additive effect with OmpA and IbeB in E. coli $\mathrm{K} 1$ penetration into the CNS. (Pediatr Res 51: 559-563, 2002)

\section{Abbreviations}

BMEC, brain microvascular endothelial cells CSF, cerebrospinal fluid

Km, kanamycin

Cm, chloramphenicol

Rif, rifampin

$\mathbf{K m}^{\mathbf{s}}$, Km susceptible

$\mathbf{C m}^{\mathbf{r}}, \mathrm{Cm}$ resistant

Rif $^{r}$, Rif resistant
The mortality and morbidity associated with neonatal meningitis remain significant despite advances in antimicrobial chemotherapy and supportive care $(1,2)$. Inadequate knowledge of the pathogenesis and pathophysiology of this disease has contributed to this poor outcome. For example, most cases of Escherichia coli meningitis develop as a result of hematogenesis spread, but it is not completely understood how circulating E. coli crosses the blood-brain barrier (3).

Our previous investigations using in vitro and in vivo models of the blood-brain barrier have indicated that successful traversal of the blood-brain barrier by circulating E. coli K1

Received August 7, 2001; November 13, 2001

Correspondence and reprint requests: Kwang Sik Kim, M.D., Division of Pediatric Infectious Diseases, Johns Hopkins University School of Medicine, 600 N. Wolfe Street, Park 256, Baltimore, MD 21287, U.S.A.; e-mail: kwangkim@jhmi.edu

Supported by National Institutes of Health grants R01-NS 26310, AI 47225, and HL 61951.

Current address (Y.W.): University of Southern California School of Dentistry, Room 4281, Los Angeles, CA 90089, U.S.A. requires 1) a high level of bacteremia and 2) invasion of BMEC (3). This was shown by the demonstration that a high degree of bacteremia is necessary but not sufficient for the development of meningitis by E. coli and that invasion of BMEC is a prerequisite for E. coli penetration into the CNS in vivo. Our previous investigations have also identified that specific microbial determinants such as OmpA, Ibe proteins, TraJ, CNF1, and AslA are required for invasion of BMEC in vitro and crossing of the blood-brain barrier in vivo (3-11), but it is unclear why these $E$. coli determinants are necessary for penetration into the CNS. The contributions of Ibe proteins, TraJ, CNF1, and AslA to invasion of BMEC have been identified by using their respective isogenic mutants of $E$. coli $\mathrm{K} 1$ strain RS218 (or E44), whereas OmpA was previously identified by using the OmpA deletion mutant, strain E91, which was constructed from strain E44 by P1 transduction (5).

In the present study, we constructed the isogenic ompA deletion mutant from the parent $E$. coli $\mathrm{K} 1$ strain E44 as well as from the $i b e B$ deletion mutant and examined their ability to 
invade BMEC in vitro and to enter the $\mathrm{CNS}$ in the newborn rat model of hematogenous meningitis in vivo.

\section{METHODS}

Bacterial strains, plasmids, and culture conditions. Bacterial strains, plasmids, and their relevant characteristics are described in Table $1(5,7,13,28,29)$. E. coli DH5 $\alpha$ (Invitrogen, Carlsbad, CA, U.S.A.) or JM101 (12) were used as a host strain for plasmids. E. coli strains were cultured at $37^{\circ} \mathrm{C}$ in Luria broth $(1 \%$ tryptone, $0.5 \%$ yeast extract, $0.5 \% \mathrm{NaCl})$ or brain heart infusion broth (Difco Laboratories, Detroit, MI, U.S.A.). When necessary, the medium was supplemented with ampicillin $(100 \mu \mathrm{g} / \mathrm{mL}), \mathrm{Km}(40 \mu \mathrm{g} / \mathrm{mL}), \mathrm{Cm}(25 \mu \mathrm{g} / \mathrm{mL})$, or Rif $(50 \mu \mathrm{g} / \mathrm{mL})$. Bacterial cultures were stored in Luria broth plus $20 \%$ glycerol at $-70^{\circ} \mathrm{C}$.

Construction of ompA deletion mutant in $\mathrm{E}$. coli. The DNA region containing ompA in E. coli $\mathrm{K} 1$ strain RS218 has been shown to be very similar to that of a K12 strain (13), so the sequence information of K12 strain (accession numbers, D90733 and U00096) was used to generate the ompA deletion DNA. A 1.06-kb DNA fragment upstream of ompA was produced by PCR with $p f u$ DNA polymerase (Stratagene, La Jolla, CA, U.S.A.) and the primers Omp1 and Omp1R (Table 2) using the RS218 genomic DNA as a template. The PCR product was digested with EcoRI and EcoRV, and cloned between the EcoRI and SmaI sites in pK194 $\left(\mathrm{km}^{\mathrm{r}}\right)$ to produce pK-omp1. Similarly, a 1.05-kb PCR fragment downstream of ompA was generated with primers Omp2 and Omp2R, and cloned in pUC13 (12) at the same sites. This cloned DNA was released and transferred into $\mathrm{pK}$-omp1 at the same sites to produce $\mathrm{pK}-\Delta o m p$. The resulting plasmid was verified by digestion with restriction enzymes BamHI, EcoRI, and HinIII, as well as by PCR using primers Omp3 and Omp3R: a 1.3-kb PCR product was obtained from the $\mathrm{pK}-\Delta o m p$ plasmid and a $2.5-\mathrm{kb}$ fragment was amplified from the chromosomal DNA of RS218. The cloned DNA in pK- $\Delta o m p$ was further verified by sequencing from both ends using the M13/pUC primers (12).

A 1.2-kb AccI-BstI fragment containing the $\mathrm{Cm}^{\mathrm{r}}$ gene was cleaved from pACYC184 (New England BioLabs) and used to replace the 100-bp AccI fragment (one of the AccI sites is on the vector) in the middle of the $\triangle o m p A$ DNA in pK- $\Delta$ omp to

Table 1. Bacterial strains and plasmids used in this study

\begin{tabular}{|c|c|c|}
\hline $\begin{array}{l}\text { Strains/ } \\
\text { plasmids }\end{array}$ & Genotype or characteristics & $\begin{array}{l}\text { Reference or } \\
\text { source }\end{array}$ \\
\hline \multicolumn{3}{|l|}{ E. coli } \\
\hline RS218 & CSF isolate, O18:K1:H7 & 5,13 \\
\hline E44 & $\operatorname{Rif}^{\mathrm{r}}$ of RS 218 & 5,13 \\
\hline E98 & $\mathrm{E} 44\left(\Delta o m p A:: \mathrm{Cm}^{\mathrm{r}}\right)$ & This study \\
\hline IB7D5 & $\mathrm{E} 44(\Delta i b e B)$ & 7 \\
\hline DK-OB & $\mathrm{E} 44\left(\Delta\right.$ ibeB,$\Delta$ omp $\left.A:: \mathrm{Cm}^{\mathrm{r}}\right)$ & This study \\
\hline SM10 גpir & $\begin{array}{l}\text { thi thr leu tonA lacY supE } \\
\text { recA }:: \mathrm{RP} 4-2-\mathrm{Tc}:: \mathrm{Mu}(\lambda \text { pir) pro endA } \\
\quad \text { hsdA hsdR supF }\end{array}$ & 28 \\
\hline \multicolumn{3}{|c|}{ 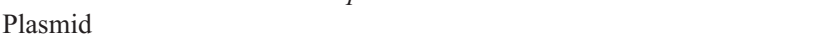 } \\
\hline pK194 & $\mathrm{Km}^{\mathrm{r}}$, lacZ' (pACYC184 derivative) & 29 \\
\hline pKE325 & $\begin{array}{l}\text { K12 ompA with its own promoter in } \\
\text { pK194 }\end{array}$ & This study \\
\hline
\end{tabular}

produce $\mathrm{pK}-\Delta o m p \mathrm{Cm}$. A $0.7-\mathrm{kb}$ DNA containing the $\mathrm{R} 6 \mathrm{~K}$ replication origin and an oriT sequence were purified from pBSL238 (14), and used to replace the 0.8-kb HindIII-XbaI fragment containing the replication origin of $\mathrm{pK}-\Delta o m p \mathrm{Cm}$. The plasmid, named $\mathrm{p} \Delta$ omp $\mathrm{Cm} 238$, was then transferred into E. coli strain E44 by conjugation as previously described $(6,7)$ using the E. coli SM10גpir as the host. The $\mathrm{Cm}^{\mathrm{r}} \mathrm{Rif}^{\mathrm{r}}$ transconjugants were selected and tested for Km resistance. Three of 60 colonies were found to be $\mathrm{Km}^{\mathrm{s}}$. These colonies were examined by PCR using Omp3 and Omp3R (Table 2) to verify the deletion of ompA. The OmpA phenotype of the mutant was verified by Western blot using polyclonal anti-OmpA antibody (5). The mutant was termed E98.

Construction of ompA and ibeB double-deletion mutant. The ompA deletion suicide plasmid $\mathrm{p} \Delta$ omp $\mathrm{Cm} 238$ was transferred into the $i b e B$ deletion mutant IB7D5 as described above. The $\mathrm{Cm}^{\mathrm{r}} \mathrm{Rif}^{\mathrm{r}}$ and $\mathrm{Km}^{\mathrm{s}}$ transconjugants were selected. The double mutant, termed DK-OB, was verified by PCR for the deletion of omp $A$ as described above, and for $i b e B$ with primers IB7-31 and IB7-35 as described previously (7).

Tissue cultures and in vitro invasion assays. BMEC were prepared from human brains, and invasion assays were performed as previously described $(4,6-8)$. Approximately $10^{7}$ bacteria in $500 \mu \mathrm{L}$ of experimental medium (Ham's F12: medium 199-1 $\times$ Earle salts, $5 \%$ heat-activated fetal bovine serum, $1 \%$ sodium pyruvate, $0.5 \%$ glutamine; Irvine Scientific, Santa Ana, CA, U.S.A.) were added to confluent BMEC with a multiplicity of infection of 100 . The monolayers were incubated for $1.5 \mathrm{~h}$ at $37^{\circ} \mathrm{C}$ to allow invasion to occur. The number of intracellular bacteria was determined after the extracellular bacteria were killed by incubation of the monolayers with experimental medium containing gentamicin $(100 \mu \mathrm{g} / \mathrm{mL})$ for $1 \mathrm{~h}$. Results were expressed as percent invasion: [100 $\times$ (number of intracellular bacteria recovered/number of bacteria incubated)].

Neonatal rat model of hematogenous E. coli K1 meningitis. E. coli bacteremia and meningitis, defined as positive CSF culture, were induced in 5-d-old rats by a previously described method $(3,8)$. Briefly, outbred, specific pathogen-free, pregnant Sprague-Dawley rats with timed conception were purchased from Charles River Breeding Laboratories (Wilmington, MA, U.S.A.); the rats delivered in our vivarium 5 to $7 \mathrm{~d}$ after they arrived. At $5 \mathrm{~d}$ of age, all members of each litter were randomly divided into four groups to receive via intracardiac injection $1.0 \times 10^{7}$ colony-forming units of the parent strain

Table 2. Oligonucleotides used in this study

\begin{tabular}{|c|c|c|c|}
\hline Primer & Sequence* & $\begin{array}{l}\text { Restriction } \\
\text { site }\end{array}$ & Location $\dagger$ \\
\hline Omp1 & 5'-ATCGaATTCAATTGTGCCCAACG & EcoRI & -1160 \\
\hline Omp1R & 5'-TCTACCGATATCTTCGGC & EcoRV & -113 \\
\hline Omp2 & 5'-CACTGgTCGACTGCCTGGCTCC & SalI & +956 \\
\hline Omp2R & 5'-TGCAaAGCTTTGTAGCGTACGCG & HindIII & +1984 \\
\hline Omp3 & 5'-GTACACTTCAGGCTATGC & None & -864 \\
\hline Omp3R & 5'-CGGTAACATTGAGATCGC & None & +1571 \\
\hline
\end{tabular}

* The restriction site is underlined; the lower case letter indicates the mutant nucleotide.

$\dagger$ The first nucleotide of the ompA open reading frame is designated as +1 . 
E44, strain E98 $\left(\Delta o m p A:: \mathrm{Cm}^{\mathrm{r}}\right)$, strain IB7D5 $(\Delta i b e B)$, or strain DK-OB $\left(\Delta i b e B, \Delta o m p A:: \mathrm{Cm}^{\mathrm{r}}\right)$. Approximately 1 to $2 \mathrm{~h}$ after bacterial inoculation, blood and CSF specimens were obtained as described previously for quantitative cultures $(3,8)$. This study was approved by the Institutional Animal Care and Use Committee.

Nucleotide sequence accession number. The sequence of ompA from E. coli $\mathrm{K} 1$ strain RS218 has been submitted to the GenBank database under accession number AF234269.

\section{RESULTS}

Construction of the isogenic $\Delta$ ompA mutant of E. coli $K 1$. The $E$. coli $\mathrm{K} 12$ ompA gene has been shown to hybridize with the ompA DNA of the K1 strain RS218, and their restriction maps were similar by Southern hybridization (13). Therefore, the $E$. coli $\mathrm{K} 12$ DNA information was used to obtain the E. coli $\mathrm{K} 1$ ompA DNA by PCR. The nucleotide sequence of the K1 ompA gene revealed that, compared with the E. coli $\mathrm{K} 12$ ompA, 20 of 1038 nucleotides differed between the two open reading frames (98\% identities). However, only three of the 325 deduced amino acid residues differ between K1 and K12 OmpA: one is in the $\beta$-barrel of OmpA, Ile93 (K12 strain) versus Val (K1 strain); two are in the periplasmic domain, Asn203 versus Asp, and Gly251 versus Ala.

The omp $A$ deletion mutant, strain E91, used in our previous studies (5) was constructed from E44 by P1 transduction using the E. coli $\mathrm{K} 12$ strain BRE51 ( $\Delta$ sulA-ompA) containing a tetracycline-resistance gene at or near the ompA locus as the donor (15) and therefore, is not an isogenic ompA deletion mutant of E44. Here, we constructed an isogenic ompA deletion mutant from E44. Three $\mathrm{Cm}^{\mathrm{r}} \mathrm{Km}^{\mathrm{s}}$ transconjugants were isolated, and the lack of omp $A$ in these colonies was verified by PCR with primers Omp3 and Omp3R. Figure $1 A$ shows that a 2.5-kb DNA was amplified from E44 (lane 6) whereas a 2.6-kb amplicon was produced in the deletion mutants owing to the replacement of ompA with the $\mathrm{Cm}^{\mathrm{r}}$ gene (lanes 2-4). Both DNA fragments were amplified from a $\mathrm{Cm}^{\mathrm{r}}$ colony that resulted from a single crossover of $\mathrm{p} \Delta$ omp $\mathrm{Cm} 238$ (lane 5). OmpA-negative phenotype in the mutant was also verified by
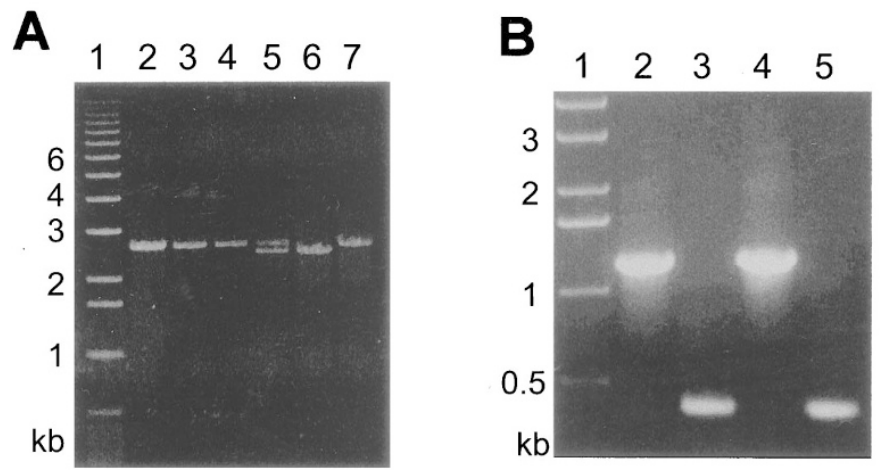

Figure 1. $A, \mathrm{PCR}$ amplification of the ompA gene locus in the deletion mutants. Lane 1, 1-kb ladder; lanes 2-4, amplicons from three ompA deletion mutants; lane 5, from single-crossover mutant; lane 6, from E44; lane 7, from $\mathrm{p} \Delta$ ompCm238. B, PCR examination of the $\Delta i b e B$ gene locus in E44 and its derivatives. Lane 1, 1-kb ladder; lane 2, PCR DNA from E44; lane 3, from IB7D5; lane 4, from E98; lane 5, from DK-OB.
Western blot with the anti-OmpA antibodies as previously described (5). The mutant was named E98, and its colony morphology and growth characteristics were identical to those of strain E44. The biochemical reactions were identical between E44 and E98 on the Vitek Gram-negative Identification+ Card (BioMerieux Vitek, Inc., Charbonnieres les Bains, France).

Similarly, an isogenic ompA deletion mutant was constructed from strain IB7D5 ( $\triangle$ ibeB $)$, and the lack of OmpA was verified by PCR and Western blot as described above (not shown). As described previously, strain IB7D5 is an isogenic in-frame $i b e B$ deletion mutant of E44 (7). Thus, the ompA deletion mutant of strain IB7D5 represents an isogenic deletion of both ompA and $i b e B$, which was named DK-OB. The absence of $i b e B$ was verified by PCR with primers IB7-31 and IB7-35 (7) (Fig. 1B). A 1.2-kb amplicon was produced in either E44 (lane 2) or E98 (lane 4), whereas a 346-bp DNA was synthesized in both $\Delta i b e B$ mutants (lanes 3 and 5).

Noninvasive phenotype of the ompA and ibeB deletion mutants. We have previously shown that the ompA-negative mutant, E91, and the ibeB deletion mutant, IB7D5, were significantly less invasive in BMEC in vitro compared with the parent strain $(5,7)$. The newly constructed isogenic mutant, E98, was also shown to be significantly less invasive in BMEC than the parent strain E44 (Table 3). The plasmid pKE325 was constructed, which contained the E. coli $\mathrm{K} 12 \mathrm{omp} A$ gene with its promoter. This plasmid was able to complement strain E98 for BMEC invasion to the level of the parent strain with the vector, i.e. $\mathrm{E} 44$ (pK194; Table 3). These findings indicate that OmpA is indeed required for E. coli $\mathrm{K} 1$ invasion of BMEC. As expected, the $i b e B$ deletion mutant, IB7D5, was less invasive in BMEC compared with the parent strain, E44. Of interest, when the ompA/ibeB double knockout, DK-OB, was examined for its ability to invade BMEC compared with its individual omp $A$ and $i b e B$ deletion mutants, its invasion capacity did not differ significantly from that of single-gene deletion mutants (Table 3).

Prevalence of meningitis in infant rats. We next examined the single and double knockouts for their ability to invade the CNS in our well-established infant rat model of experimental hematogenesis meningitis. As shown in Table 4, the magnitudes of bacteremia were similar among the four groups of animals. However, the occurrence of meningitis (defined as positive CSF cultures) was significantly lower $(p<0.05)$ in

Table 3. In vitro BMEC invasive phenotype of E44 and its derivatives

\begin{tabular}{llc}
\hline & \multicolumn{1}{c}{ Strain (characteristics) } & Invasion frequency $(\%) *$ \\
\hline Exp. 1 & E44 & $0.104 \pm 0.025$ \\
& HB101 & $0.008 \pm 0.001$ \\
& E98 $(\Delta$ ompA $:: \mathrm{Cm})$ & $0.031 \pm 0.003 \dagger$ \\
& IB7D5 $(\Delta i b e B)$ & $0.031 \pm 0.011 \dagger$ \\
& DK-OB $(\Delta i b e B, \Delta$ omp $A:: \mathrm{Cm})$ & $0.026 \pm 0.012 \dagger$ \\
Exp. 2 & E44 (pK194) & $0.080 \pm 0.026$ \\
& E98 (pK194) & $0.018 \pm 0.006 \dagger$ \\
& E98 (pKE325) & $0.083 \pm 0.02$ \\
\hline
\end{tabular}

* All invasion experiments were conducted in triplicate and performed three times. Results are expressed as mean $\pm \mathrm{SD}$.

$\dagger$ Significantly lower than E44 $(p<0.01)$.

$\$$ Significantly lower than E44 (pK194) or E98 (pKE325) $(p<0.01)$. 
animals receiving the $\triangle$ ompA mutant, E98 (six of 22, or $27 \%$ ), and the $\Delta i b e B$ mutant, IB7D5 (five of 21 , or $24 \%$ ), than in those receiving the parent strain, E44 (12 of 19, or 63\%). These findings with the $\Delta i b e B$ mutant, IB7D5, were similar to those of our previous study with the noninvasive mutant 7A-33 with a single TnphoA insertion in $i b e B$, in which we showed that the mutant 7A-33 was significantly less able to penetrate the CNS in vivo than its parent strain (7). Consistent with the in vitro BMEC phenotype, the prevalence of meningitis in 5-d-old rats infected with the $\triangle o m p A, \Delta i b e B$ mutant did not differ significantly from that of animals infected with the $\triangle o m p A$ or $\triangle i b e B$ mutant. It is also important to point out that the BMEC invasion frequency of $0.1 \%$ for E. coli $\mathrm{K} 1$ strain E44 (Table 3) is related to enhanced invasion of the CNS in vivo (Table 4).

\section{DISCUSSION}

OmpA is a major outer membrane protein in E. coli and is highly conserved among Gram-negative bacteria. We have previously demonstrated a novel phenotype of OmpA, i.e. its contribution to $E$. coli $\mathrm{K} 1$ invasion of BMEC in vitro (5). This was shown by the demonstration that 1 ) the invasive capability of the nonisogenic ompA deletion mutant, E91, was restored to the level of the parent $E$. coli $\mathrm{K} 1$ strain by complementation with the E. coli $\mathrm{K} 12$ ompA gene, and 2) OmpA proteins and anti-OmpA antibodies inhibited the invasion of OmpApositive E. coli into BMEC. In the present study using an isogenic ompA-deleted mutant, we verified the role of OmpA in E. coli $\mathrm{K} 1$ invasion of $\mathrm{BMEC}$, i.e. the isogenic ompA deletion mutant was less invasive in BMEC than its parent $E$. coli $\mathrm{K} 1$ strain and its invasive ability was restored to the level of the parent strain by complementation with the E. coli $\mathrm{K} 12$ ompA gene. Our sequencing analysis showed the E. coli $\mathrm{K} 1$ and K12 OmpAs are essentially identical. In addition, the isogenic ompA deletion mutant was significantly less able to cross the blood-brain barrier compared with the parent strain in our hematogenous meningitis animal model. These findings indicate that OmpA is indeed a critical determinant for $E$. coli $\mathrm{K} 1$ penetration into the CNS in vivo.

We have previously identified that OmpA and other E. coli factors contribute to the ability of $E$. coli $\mathrm{K} 1$ to cross the blood-brain barrier in vivo, which include Ibe proteins, TraJ, CNF1, and AslA (4-11). We and others have also shown that these $E$. coli $\mathrm{K} 1$ genes are located at different regions of the $E$. coli $\mathrm{K} 1$ genome $(3,16,17)$. It is unclear how different invasive

Table 4. Development of bacteremia and meningitis (defined as positive $C S F$ cultures) in four groups of newborn rats receiving $E$.

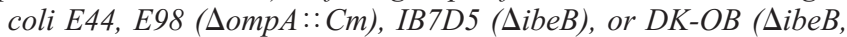
$\triangle o m p A:: C m)$

\begin{tabular}{lccc}
\hline $\begin{array}{c}\text { E. coli } \\
\text { strain }\end{array}$ & $\begin{array}{c}\text { No. of } \\
\text { animals }\end{array}$ & $\begin{array}{c}\text { Bacteria (log CFU/mL } \\
\text { of blood, mean } \pm \text { SD) }\end{array}$ & $\begin{array}{c}\text { No. (\%) of animals } \\
\text { with positive CSF }\end{array}$ \\
\hline E44 & 19 & $7.18 \pm 0.63$ & $12(63)$ \\
E98 & 22 & $7.05 \pm 0.49$ & $6(27)^{*}$ \\
IB7D5 & 21 & $7.29 \pm 0.69$ & $5(24)^{*}$ \\
DK-OB & 22 & $6.95 \pm 0.37$ & $6(27)^{*}$ \\
\hline
\end{tabular}

* Significantly $(p<0.05)$ fewer than animals receiving E44.

$\mathrm{CFU}$, colony-forming units. loci of E. coli $\mathrm{K} 1$ contribute to crossing of the blood-brain barrier.

Our previous investigations have revealed that E. coli $\mathrm{K} 1$ translocates from blood to the CNS without altering the integrity of the blood-brain barrier and transcytosis of BMEC by $E$. coli $\mathrm{K} 1$ occurs without any change in the integrity of monolayers $(2,18)$. Transmission election microscopy revealed that E. coli $\mathrm{K} 1$ invades BMEC via a zipperlike mechanism and transmigrates through BMEC in an enclosed vacuole without intracellular multiplication (19). E. coli $\mathrm{K} 1$ invasion of BMEC requires actin cytoskeletal rearrangements and induces tyrosine phosphorylation of focal adhesion kinase and paxillin, which are thought to be related to OmpA-BMEC interactions (20). In this regard, it is important to note that OmpA interaction with the BMEC GlcNAc $\beta 1-4$ GlcNAc epitopes was shown to contribute to the traversal of $E$. coli $\mathrm{K} 1$ across the blood-brain barrier and a specific receptor for OmpA was demonstrated to be present only on BMEC (21).

Our recent studies demonstrate that ibeA encodes an outer membrane protein with three transmembrane domains, which binds to a novel $45-\mathrm{kD}$ receptor on BMEC, named Ibe10R (6, 22). The addition of the Ibe10R protein or a polyclonal antibody raised against Ibe10R significantly inhibits $E$. coli $\mathrm{K} 1$ invasion of BMEC in vitro (16). These findings suggest that OmpA and IbeA contribute to BMEC invasion via ligandreceptor interactions. IbeB also displays the characteristics of outer membrane proteins (7), suggesting that the IbeBmediated invasion of BMEC may also occur via a ligandreceptor interaction. We therefore speculate that OmpA and Ibe proteins might be additive or synergistic in their contributions to $E$. coli $\mathrm{K} 1$ invasion of BMEC. Because ompA and $i b e B$ have $\mathrm{K} 12$ homologs $(5,6)$ and are more prevalent in E. coli $\mathrm{K} 1$ isolates from CSF compared with ibeA (7), we examined the roles of OmpA and IbeB deletions in E. coli $\mathrm{K} 1$ invasion of BMEC in vitro and traversal of the blood-brain barrier in vivo. We found that $\triangle o m p A$ and $\triangle i b e B$, alone and in combination, are, as expected, significantly less invasive in BMEC both in vitro and in vivo compared with the parent strain. We emphasize that the BMEC invasion frequency of the parent strain (approximately $0.1 \%$ ) is considerably lower than the reported invasion frequency of epithelial cells by other Gram-negative bacteria such as Salmonella and Shigella species (usually 1 to $10 \%)$, but as shown here and in our previous publications $(6,8$, 9 ), the BMEC invasion frequency of approximately $0.1 \%$ relates to enhanced bacterial penetration through the bloodbrain barrier and thus is biologically relevant. Our unexpected finding, however, was that the invasion phenotypes of the $\triangle o m p A, \triangle i b e B$ mutant were similar to those of the single-gene deletion mutants both in vitro and in vivo.

The reasons for no additive or synergistic effect observed with the $\triangle$ ompA, $\triangle i b e B$ mutant are not clear. We and others have previously shown that $\mathrm{S}$ fimbriae consisting of the major subunit $\mathrm{SfaA}$ and minor subunits including SfaS are one of the major structures contributing to $E$. coli binding to BMEC. The two binding domains of $\mathrm{S}$ fimbriae have been identified, i.e. $\mathrm{SfaS}$ adhesin for binding to BMEC sialoglycoproteins and SfaA for binding to BMEC sulfated glycolipid (sulfatide) (23, 24). Inasmuch as $\mathrm{SfaS}$ is shown to be preferentially located at 
the tip of S fimbriae (25), we speculate that the critical binding of $\mathrm{S}$ fimbriated E. coli to BMEC occurs via SfaS-BMEC sialoglycoprotein interaction, which is followed by an association of bacteria with sulfatide for a more intimate contact of organism to BMEC to withstand blood flow in vivo; such a binding is probably required for subsequent crossing of the blood-brain barrier. E. coli K1 strain RS218 (or E44) was found to possess $\mathrm{S}$ fimbriae, which was maintained in the $\triangle o m p A, \Delta i b e B$ mutants; thus, it is unlikely that their bindings to BMEC via $\mathrm{S}$ fimbriae are affected in these mutants. As described above, we anticipate finding that OmpA and IbeB interact with different receptors on BMEC, for their invasion and thus the effects of OmpA and IbeB on BMEC invasion will be at least additive. We have previously shown that $E$. coli $\mathrm{K} 1$ invasion of BMEC requires host cell actin cytoskeletal rearrangements, as shown by invasive E. coli $\mathrm{K} 1$-associated Factin condensation and blockade of invasion by the microfilament-disrupting agent, cytochalasin D (19). In addition, we have shown that $E$. coli invasion of BMEC requires several signal transduction pathways such as focal adhesion kinase, phosphatidylinositol 3-kinase, and cytosolic phospholipase $\mathrm{A}_{2}$ $(20,26,27)$. It remains speculative whether OmpA and IbeB interactions with their receptors involve common signaling pathways contributing to host cell actin cytoskeletal rearrangements and invasion of BMEC, thereby exhibiting no additive effects with $\triangle o m p A$ and $\triangle i b e B$. An alternative explanation may be that the interaction of OmpA or IbeB with its respective receptor hinders the other ligand-receptor interactions, thus eliminating any additive effect. Identification and characterization of specific BMEC receptors for OmpA and Ibe B should help clarify the observation described in this report.

Acknowledgments. The authors thank C. Wass for technical assistance, S.H. Huang for providing the strain IB7D5, M. Alexeyev of the University of Alabama for providing the plasmid pBSL238, and the Clinical Microbiology Laboratory of CHLA for testing E. coli strains with the Vitek system.

\section{REFERENCES}

1. Uhanand M, Mustafa MM, McCracken GH, Nelson JD 1993 Gram-negative enteric bacillary meningitis: a twenty-year experience. J Pediatr 122:15-21

2. Dawson KG, Emerson JC, Burns JL 1999 Fifteen years of experience with bacterial meningitis. Pediatr Infect Dis J 18:816-22

3. Kim KS 2001 E. coli translocation at the blood-brain barrier. Infect Immun 69:52175222

4. Kim KS, Itabashi H, Gemski P, Sadoff J, Warren RL, Cross AS 1992 The K1 capsule is the critical determinant in the development of Escherichia coli meningitis in the rat. $\mathrm{J}$ Clin Invest 90:897-905

5. Nemani P, Wass C, Stins MF, Weiser J, Huang SH, Kim KS 1996 Outer membrane protein A of E. coli contributes to invasion of brain microvascular endothelia cells. Infect Immun 64:146-153
6. Huang SH, Wan ZS, Chen YH, Jong AY, Kim KS 2001 Further characterization of Escherichia coli brain microvascular endothelial cell invasion gene ibeA by deletion, complementation, and protein expression. J Infect Dis 183:1071-1078

7. Huang SH, Chen YH, Fu Q, Wang Y, Stins M, Wass C 1999 Identification and characterization of an $E$. coli invasion gene locus $i b e B$ required for penetration of brain microvascular endothelia cells. Infect Immun 67:2103-2109

8. Wang Y, Wang SH, Wass C, Kim KS 1999 The gene locus yijP contributes to E. coli invasion of brain microvascular endothelial cells. Infect Immun 67:4751-4756

9. Badger JL, Wass C, Weissman S, Kim KS 2000 Application of signature-tagged mutagenesis for the identification of $E$. coli $\mathrm{K} 1$ genes that contribute to invasion of the blood brain barrier. Infect Immun 68:5056-5061

10. Hoffman JA, Badger JL, Zhang Y, Huang SH, Kim KS 2000 E. coli K1 AslA contributed to invasion of brain microvascular endothelial cells in vitro and in vivo. Infect Immun 68:5062-5067

11. Wang Y, Wass C, Kim KS 2000 Cytoxic necrotizing factor 1 promotes Escherichia coli $\mathrm{K} 1$ invasion of brain microvascular endothelial cells in vitro and in vivo. Abstracts of the 100th American Society for Microbiology General Meeting, Los Angeles, Abstr B108:65

12. Vieira J, Messing J 1982 The pUC plasmids in M13mp7-derived system for insertion mutagenesis and sequencing with synthetic universal primers. Gene 19:259-268

13. Weiser JN, Gotschlich EC 1991 Outer membrane protein A (OmpA) contributes to serum resistance and pathogenicity of Escherichia coli K-1. Infect Immun 59:22522258

14. Alexeyev MF, Shokolenko IN 1995 RP4 oriT and RP4 oriT-R6K oriV DNA cassettes for construction of specialized vectors. Biotechniques 19:22-26

15. Bremer E, Silhavy TJ, Maldener M, Cole TS 1986 Isolation and characterization of mutants deleted for the sulA-ompA region of the Escherichia coli $\mathrm{K}-12$ chromosome. FEMS Microbiol Lett 33:173-178

16. Rode CK, Melkerson-Watson LJ, Johnson AT, Bloch CA 1999 Type-specific contributions to chromosome size differences in Escherichia coli. Infect Immun 19:230236

17. Bonacorsi SPP, Clermont O, Tinsley C, LeGall I, Beaudoin JC, Elion J 2000 Identification of regions of the Escherichia coli chromosome specific for neonatal meningitis-associated strains. Infect Immun 68:2096-2101

18. Stins MF, Badger JL, Kim KS 2001 Bacterial invasion and transcytosis in transfected human brain microvascular endothelial cells. Microb Pathogen 30:19-28

19. Nemani PV, Wass CA, Stins M, Shimada H, Kim KS 1999 Outer membrane protein A-promoted actin condensation of brain microvascular endothelial cells is required for $E$. coli invasion. Infect Immun 67:5775-5783

20. Reddy MA, Wass CA, Kim KS, Schlaepfer DD, Prasadarao NV 2000 Involvement of focal adhesion kinases in $E$. coli invasion of human brain microvascular endothelial cells. Infect Immun 68:6419-6422

21. Nemani PV, Wass CA, Kim KS 1996 Endothelia cell GlcNAcl-4 GlcNAc epitopes for outer membrane protein A enhance traversal of $E$. coli across the blood-brain barrier. Infect Immun 64:154-160

22. Nemani PV, Huang SH, Wass CA, Kim KS 1999 Identification and characterization of a novel Ibe10 binding protein contribution to $E$. coli invasion of brain microvascular endothelial cells. Infect Immun 67:1131-1138

23. Stins MF, Prasadarao NV, Ibric L, Wass CA, Luckett P, Kim KS 1994 Binding characteristics of S-fimbriated $E$. coli to isolated brain microvascular endothelial cells. Am J Pathol 145:1228-1236

24. Prasadarao NV, Wass CA, Hacker J, Jann K, Kim KS 1993 Adhesion of S-fimbriated Escherichia coli to brain glycolipids mediated by sfaA gene-encoded protein of S-fimbriae. J Biol Chem 268:10356-10363

25. Moch T, Hoschutzky H, Hacker J, Kroncke KD, Jann K 1987 Isolation and characterization of the $\alpha$-sialyl- $\beta$-galactosyl-specific adhesin from fimbriated Escherichia coli. Proc Natl Acad Sci USA 84:3462-3466

26. Reddy MA, Prasadarao NV, Wass CA, Kim KS 2000 Phosphatidylinositol 3-kinase activation and interaction with focal adhesion kinase in $E$. coli $\mathrm{K} 1$ invasion of human brain microvascular endothelial cells. J Biol Chem 275:36769-36774

27. Das A, Asatryan L, Reddy MA, Wass CA, Stins MF, Joshi S, Boventre JV, Kim KS 2001 Differential role of cytosolic phospholipase $\mathrm{A}_{2}$ in the invasion of brain microvascular endothelial cells by Escherichia coli and Listeria monocytogenes. J Infect Dis 184:732-737

28. Taylor RK, Manoil C, Mekalanos J 1989 Broad-host-range vectors for delivery of TnphoA: use in genetic analysis of secreted virulence determinants of Vibrio cholerae. J Bacteriol 171:1870-1878

29. Jobling MG, Holmes RK 1990 Construction of vectors with the p15a replicon, kanamycin resistance, inducible lacZ $\alpha$ and pUC18 or pUC19 multiple cloning sites. Nucleic Acids Res 18:5315-5316 